\title{
Market Participation and Sunspot Equilibria
}

\author{
YVES BALASKO \\ Universities of Geneva and Paris I \\ DAVID CASS \\ University of Pennsylvania
}

and

KARL SHELL

Cornell University

First revision received April 1988; final version accepted April 1995 (Eds.)

\begin{abstract}
We investigate the structure of competitive equilibria in an exchange economy parametrized by (i) endowments and (ii) restrictions on market participation. For arbitrary regular endowments, if few consumers are restricted, there are no sunspot equilibria. If endowments are allowed to vary, while restrictions on market participation are fixed, there is a generic set of preferences such that sunspot equilibria exist for a non-empty subset of endowments. Our analysis extends to the general case of an arbitrary number of restricted consumers the results of Cass and Shell for the polar cases in which either (i) no consumers are restricted or (ii) all consumers are restricted.
\end{abstract}

\section{INTRODUCTION}

In actual economies, there are restrictions on market participation. Some people are able to trade on a given market, while others are not. Some of the restrictions on market participation are based on law or custom. For example, it is against the law in some countries to sell alcoholic beverages to minors. In these countries, minors are restricted from participating in the wine and beer markets. Other restrictions are inherent in the definition of the market. For example, an actual market "meets" at some particular time. As a consequence, only people alive and present at that time can trade on the market. All others (including the yet unborn) are restricted from participating on the market.

A model of restricted market participation is developed in Section 2. It is essentially the original Cass-Shell (1983) pure-exchange sunspot model, but alterations have been made to permit continuous variations in market participation. The number of commodities is finite. Consumers maximize von Neumann-Morgenstern utility functions. There is no intrinsic uncertainty. There are two possible (extrinsic) states states of nature. Markets are complete, but participation on the securities market is possibly restricted. In Section 3 , we analyse consumer behaviour and equilibrium outcomes as a function of market participation while endowments remain fixed at some regular value. There are $m$ types of consumers, with a continuum of individuals of each type. The economy is parameterized by the vector $\lambda=\left(\lambda_{1}, \ldots, \lambda_{h}, \ldots, \lambda_{m}\right)$, where $\left(1-\lambda_{h}\right)$ is the "proportion" of consumers of type $h$ who are restricted from participating in the securities or financial markets. It is known that for $\lambda=0$, the fully unrestricted case, there are no sunspot equilibria: see Cass 
and Shell (1983), and Balasko (1983). for regular economies, we generalize this result: if $\lambda$ is small i.e., if relatively few consumers are restricted, then there are no sunspot equilibria. In Section 4, we analyse, for a fixed vector $\lambda$ representing proportions of agents whose participation is restricted, the existence of sunspot equilibria as a function of the endowments. It is known that if the endowments are Pareto optimal, then there are no sunspot equilibria: see again Cass and Shell (1983). We show that if the endowment vector is close to the set of Pareto-optimal allocations, then again there are no sunspot equilibria. It is also known from Cass and Shell (1983) that for $\lambda=1$, the fully-restricted case, sunspot equilibria exist if and only if there are multiple, non-sunspot equilibria. We extend the sufficiency part of this result to the case of more general market participation. Hold $\lambda$ fixed at any value but unity and assume that preferences are such that there are multiple non-sunspot equilibria for some endowments, a property that is satisfied for a generic set of preferences. Then, we show that there exist endowments for which sunspot equilibria exist.

The research reported in this paper deals with aspects of sunspot equilibria resulting from restrictions that refer to an absolute ban in market participation arising, for example, from considerations of actual presence on a particular market. This approach must be contrasted with alternative approaches that focus on the restrictions that arise from, for example, considerations of moral hazard in fulfilling commitments. These approaches have led to the study of sunspot equilibria in models of imperfect financial markets for which we refer to Cass (1992) and the mini-symposium issue of Economic Theory.

It must be stressed that the results in the present paper are based on a model in which the role of time is only implicit. Our comparative statics consists in the analysis of how changes in a few parameters, namely the vector of proportions of restricted consumers and initial endowments, affect sunspot and non-sunspot equilibria. It is a different exercise - often a very different exercise - to analyse the effects of changing the same parameters on the stationary (or even periodic) sunspot or non-sunspot equilibria in some fully articulated (infinite-horizon) dynamic model. See in that regard the literature on "comparisons of steady states" in dynamic sunspots models exemplified by Azariadis and Guesnerie (1986) and Grandmont (1989).

\section{THE PURE EXCHANGE SUNSPOT MODEL: DEFINITIONS, ASSUMPTIONS AND NOTATION}

We consider pure exchange economies with $l$ commodities and two states of nature, denoted $\alpha$ and $\beta$, and having probabilities $\pi(\alpha)$ and $\pi(\beta)$. (Therefore, there exists $2 l$ contingent commodities.) There are $m$ households or consumers. We denote by $H$ the set of consumers. The preferences of consumer $h \in H$ are defined by way of a Von NeumannMorgenstern utility function taking the form

$$
u_{h}\left(x_{h}(\alpha), x_{h}(\beta)\right)=\pi(\alpha) v_{h}\left(x_{h}(\alpha)\right)+\pi(\beta) v_{h}\left(x_{h}(\beta)\right) .
$$

The functions $u_{h}$ and $v_{h}$ are assumed to be concave besides satisfying the standard assumptions of smooth consumer theory. (See, e.g., Balasko (1988)).

Let $\Omega=\left(\mathbb{R}^{21}\right)^{m}$ denote the set of endowments of the $m$ consumers. We also consider the set $\bar{\Omega}$ consisting of symmetric endowments, i.e., $\omega \in \bar{\Omega}$ is equivalent to $\omega_{h}(\alpha)=\omega_{h}(\beta)=$ $\bar{\omega}_{h}$ for $h=1,2, \ldots, m$. Note that the set $\bar{\Omega}$ can be identified with $\mathbb{R}^{i m}$.

The price vector $p=(p(\alpha), p(\beta))$ is not normalized (in order to avoid any artificial breach in the symmetry of the model). Let $\bar{S}=\mathbb{R}_{++}^{l}$. Then $S=\bar{S} \times \bar{S}$ denotes the set of strictly positive (non-normalized) prices. 


\section{The unrestricted consumer}

The main feature of the sunspot model is that consumer $h$ is facing one or two budget constraints, depending on whether he has or has not access to an insurance market whose role is to transfer wealth across states of nature.

If consumer $h$ is unrestricted, he then faces only one budget constraint equal to

$$
p(\alpha) \cdot x_{h}(\alpha)+p(\beta) \cdot x_{h}(\beta)=(p(\alpha)+p(\beta)) \cdot \bar{\omega}_{h}=w_{h} .
$$

The demand $f_{h}\left(p(\alpha), p(\beta), w_{h}\right)$ of the unrestricted consumer $h$ is the standard Walrasian individual demand function. The individual excess demand is denoted by $z\left(p, \bar{\omega}_{h}\right)=$ $\left.f_{h}(p(\alpha), p(\beta), p(\alpha)+p(\beta)) \cdot \bar{\omega}_{h}\right)-\left(\bar{\omega}_{h}, \bar{\omega}_{h}\right)$.

From the restricted consumer to the fictitious consumers.

If consumer $h$ is restricted, then he faces two separate budget constraints, namely

$$
\left.\begin{array}{l}
p(\alpha) \cdot x_{h}(\alpha)=p(\alpha) \cdot \bar{\omega}_{h}, \\
p(\beta) \cdot x_{h}(\beta)=p(\beta) \cdot \bar{\omega}_{h} .
\end{array}\right\}
$$

This maximization problem decomposes into two separate problems, namely "to maximize $v_{h}\left(x_{h}(\alpha)\right)$ subject to $p(\alpha) \cdot x_{h}(\alpha)=p(\alpha) \cdot \bar{\omega}_{h}=w_{h}(\alpha)$ " and "to maximize $v_{h}\left(x_{h}(\beta)\right)$ subject to $p(\beta) \cdot x_{h}(\beta)=p(\beta) \cdot \bar{\omega}_{h}=w_{h}(\beta)$ "'.

Let $\bar{f}_{h}$ denote the (Walrasian) demand of a fictitious consumer $h$ whose preferences are defined in the commodity space $\mathbb{R}^{\prime}$ through the utility function $v_{h}: \mathbb{R}^{\prime} \rightarrow \mathbb{R}$ and who faces only the standard (and therefore unique) budget constraint with respect to the commodity space $\mathbb{R}^{\prime}$. Then the demand function of the restricted consumer is related to the demand of the fictitious consumer as follows.

$$
f_{h}\left(p(\alpha), p(\beta), w_{h}(\alpha), w_{h}(\beta)\right)=\left(\bar{f}_{h}\left(p(\alpha), w_{h}(\alpha)\right), \bar{f}_{h}\left(p(\beta), w_{h}(\beta)\right)\right) .
$$

We also find it convenient to consider the individual excess demand function

$$
\bar{z}_{h}\left(\bar{p}, \bar{\omega}_{h}\right)=\bar{f}_{h}\left(\bar{p}, \bar{p} \cdot \bar{\omega}_{h}\right)-\bar{\omega}_{h}
$$

of the fictitious consumer.

\section{Symmetric individual demand and prices}

It turns out that the symmetric individual demand of consumer $h$, i.e.,

$$
x_{h}=\left(x_{h}(\alpha), x_{h}(\beta)\right),
$$

where $x_{h}(\alpha)=x_{h}(\beta)$, for a price vector $p=(p(\alpha),(\beta))$, is independent of whether the consumer $h$ is restricted or unrestricted. This is described precisely in the following lemma.

Lemma 2.1. The individual demand $x_{h}=\left(x_{h}(\alpha), x_{h}(\beta)\right)$ is symmetric if the price vector $p=(p(\alpha), p(\beta))$ is equal to $(\pi(\alpha) \bar{p}, \pi(\beta) \bar{p})$ for some price vector $\bar{p} \in \bar{S}$ and, then, $\bar{x}_{h}=$ $x_{h}(\alpha)=x_{h}(\beta)=\bar{f}_{h}\left(\bar{p}, \bar{p} \cdot \bar{\omega}_{h}\right)$.

Note that the above relationship does not depend on whether consumer $h$ is restricted or not.

Proof. The property stated in Lemma 1 is obvious if $h$ is restricted. In the case of the unrestricted consumer, it follows from the strict-concavity of the utility function 
$u_{h}\left(x_{h}(\alpha), x_{h}(\beta)\right)=\pi(\alpha) v_{h}\left(x_{h}\left(x_{h}(\alpha)\right)+\pi(\beta) v_{h}\left(x_{h} \beta\right)\right)$. The reasoning goes as follows. All one has to prove is the equality

$$
x_{h}=f_{h}\left(\pi(\alpha) \bar{p}, \pi(\beta) \bar{p},(\pi(\alpha) \bar{p}+\pi(\beta) \bar{p}) \cdot \bar{\omega}_{h}\right)=\left(\bar{f}_{h}\left(\bar{p}, \bar{p} \cdot \bar{\omega}_{h}\right), \bar{f}_{h}\left(\bar{p}, \bar{p} \cdot \bar{\omega}_{h}\right)\right) .
$$

Suppose that $x_{h}=\left(x_{h}(\alpha), x_{h}(\beta)\right)$ maximizes $\left.\pi(\alpha) v_{h}(\alpha)\right)+\pi(\beta) v_{h}\left(x_{h}(\beta)\right)$ subject to the constaint $\bar{p} \cdot\left(\pi(\alpha) x_{h}(\alpha)+\pi(\beta) x_{h}(\beta)\right)=\bar{p} \cdot \bar{\omega}_{h}$. It is symmetric for the following reason. Assume the contrary: the symmetric allocation $(\bar{x}, \bar{x})$, where $\bar{x}=\pi(\alpha) x_{h}(\alpha)+\pi(\beta) x_{h}(\beta)$, satisfies the budget constraint and, given the strict concavity of the utility function $v_{h}$, also satisfies $v_{h}(\bar{x})>\pi(\alpha) v_{h}\left(x_{h}(\alpha)\right)+\pi(\beta) v_{h}\left(x_{h}(\beta)\right)$ because $x_{h}(\alpha) \neq x_{h}(\beta)$. This inequality contradicts the definition of $\left(x_{h}=\left(x_{h}(\alpha), x_{h}(\beta)\right)\right.$ as the solution of the maximization problem of consumer $h$. Therefore, $\bar{x}_{h}=x_{h}(\alpha)=x_{h}(\beta)$ maximizes $v_{h}\left(\bar{x}_{h}\right)$ subject to the constraint $\bar{p} \cdot \bar{x}_{h}=\bar{p} \cdot \overline{\boldsymbol{\omega}}_{h}$, and conversely. $\|$

\section{From restricted consumers to quasi-Walrasian consumers}

Consider the excess demand of the restricted consumer $h$

$$
\left.\left(z_{h}\left(p(\alpha), \omega_{h}\right)\right), z_{h}\left(p(\beta), \omega_{h}\right)\right) \in \mathbb{R}^{2 l} .
$$

Each expression $\bar{z}_{h}\left(p(\alpha), \bar{\omega}_{h}\right)$ and $\bar{z}_{h}\left(p(\beta), \bar{\omega}_{h}\right)$ is the excess demand of some fictitious consumer $h$ for the price vectors $p(\alpha)$ and $p(\beta)$, respectively. One can therefore introduce the quasi-Walrasian consumers $h(\alpha)$ and $h(\beta)$ where the excess demand of $h(\alpha)$ is $\left(\bar{z}_{h}\left(p(\alpha), \bar{\omega}_{h}\right), 0\right)$ while $h(\beta)$ 's excess demand is $\left(0, \bar{z}_{h}\left(p(\beta), \bar{\omega}_{h}\right)\right)$. Quasi-Walrasian consumers are studied in Appendix A. Here, it suffices to say that each of them faces a single budget constraint, the modifier "quasi" expressing the fact the consumption space has dimensionality strictly smaller than that of the underlying consumption space. For the quasi-Walrasian consumers, endowments belong to and preferences are defined on the smaller consumption space. One reason for introducing this terminology takes its origin in the emphasis put by smooth consumer theory on having all commodities as arguments of the utility function which, in our terminology, would correspond to a Walrasian consumer. Therefore a quasi-Walrasian consumer can be regarded as being a Walrasian consumer with a smaller consumption space. Another reason that justifies the use of specific terminology to distinguish quasi-Walrasian consumers from Walrasian ones is to remind us that these consumers are fictitious and that a pair of them represents a single, real consumer whose participation in financial markets is restricted. One can substitute for every restricted consumer $h$ (that has to face therefore two budget constraints) a pair of quasi-Walrasian consumers $h(\alpha)$ and $h(\beta)$

Remark. Incorporating quasi-Walrasian consumers in the general equilibrium model is not new. For example, Arrow and Hahn's (1971) study of the existence of equilibria encompasses the case of quasi-Walrasian consumers. The overlapping-generations model offers another example of quasi-Walrasian consumers, their life span being finite compared to the infinite duration of the economy. One readily sees (cf. Appendix A) that most proofs of the standard model can easily be adapted to accommodate quasi-Walrasian consumers. In other words, the properties of the general equilibrium model with quasiWalrasian consumers are essentially the same as those of the same model with only Walrasian consumers. In particular, we will make crucial use of the structure of the set of Pareto optima as a set parametrized by the utility levels of every consumer but one (for fixed total resources), a property that remains true in the setup of quasi-Walrasian consumers. 


\section{The sunspot model reformulated}

Substituting two quasi-Walrasian consumers for a consumer facing two budget constraints yields a model that is very similar to a Walrasian pure exchange economy. Specific features in terms, for example, of the symmetries of the model require, however, treatment of their own.

Consumer $h(\alpha)$ is defined as follows: the consumption space is $\mathbb{R}^{\prime} \times\{0\}$, the preferences on $\mathbb{R}^{\prime} \times\{0\}$ (identified with $\mathbb{R}^{\prime}$ ) are defined by the utility function $v_{h}$ while the initial endowments are $\omega_{h}(\alpha)=\left(\bar{\omega}_{h}, 0\right)$. A parallel formulation with $\beta$ instead of $\alpha$ and $\{0\} \times \mathbb{R}^{\prime}$ instead of $\mathbb{R}^{\prime} \times\{0\}$ defines consumer $h(\beta)$. Let $z_{h(\alpha)}$ and $z_{h(\beta)}$ denote the individual excess demand functions of these consumers $h(\alpha)$ and $h(\beta)$. Using previous notation, we can write

$$
z_{h(\alpha)}\left(p(\alpha), p(\beta), \bar{\omega}_{h}\right)=\left(\bar{z}_{h}\left(p(\alpha), \bar{\omega}_{h}\right), 0\right)
$$

and

$$
z_{h(\beta)}\left(p(\alpha), p(\beta), \bar{\omega}_{h}\right)=\left(0, \bar{z}_{h}\left(p(\beta), \bar{\omega}_{h}\right)\right)
$$

Consequently, from now on, instead of considering economies consisting of consumers facing one or two budget constraints, we are going to consider only economies consisting of Walrasian and quasi-Walrasian consumers. The quasi-Walrasian consumers are, by construction, not symmetric but, in the models we are dealing with, substituting $\beta$ for $a$ will amount to exchanging the quasi-Walrasian consumers between one another so that the economy as a whole remains symmetric with respect to $\alpha$ and $\beta$.

\section{Sunspot equilibria}

The simplest way to define a sunspot equilibrium is to consider the equilibrium allocation $x=(x(\alpha), x(\beta))$ where $x(\alpha)=\left(x_{h}(\alpha)\right) h$ and $x(\beta)=\left(x_{h}(\beta)\right)_{h}$. Such an equilibrium allocation is associated with some price vector $p=(p(\alpha), p(\beta))$. Then,

Definition 2.2. The price vector $p=(p(\alpha), p(\beta))$ is a sunspot equilibrium price vector if the corresponding equilibrium allocation $x=(x(\alpha), x(\beta))$ satisfies $x(\alpha) \neq x(\beta)$.

In other words, a sunspot equilibrium allocation is the same thing as an asymmetric equilibrium allocation. It follows from Cass and Shell (1983) and Balasko (1983)-see also Lemmas 2 and 3 below-that if there is no quasi-Walrasian agent (i.e., market participation is not restricted), then there exists no sunspot equilibrium. On the other hand, if no consumer is purely Walrasian (because of the lack of, e.g., insurance markets) and if $n$ denotes the number of non-sunspot equilibria, there exist $n^{2}-n$ sunspot equilibria. These results suggest that the extent of the restrictions on market participation is likely to play a key role in the existence of sunspot equilibria.

\section{VARIABLE RESTRICTIONS ON MARKET PARTICIPATION}

\section{Modeling variable restriction}

The concept of the extent of restricted market participation is defined within the setup of economies with fixed types of consumers by having a variable number of agents within each type. More specifically, one associates three different types of consumers with a given pair of preferences and initial endowments: the Walrasian type (simply denoted by the 
subscript $h$ ) and two quasi-Walrasian types (denoted by the subscripts $h(\alpha)$ and $h(\beta)$, respectively). Therefore, starting with $m$ pairs defined by given preferences and initial endowments, the $m$ consumers whose status with respect to market participation may vary yield an economy consisting of $3 m$ types of consumers. Consider now $k$ replicas of this economy. Let $k(h)$ be the number of those replicas for which the $h$-th consumer cannot participate in every market; the economy then consists of $k-k(h)$ consumers of type $h$ and of $k(h)$ consumers of type $h(\alpha)$ and $h(\beta)$, respectively. The equilibrium equation of this replicated economy becomes

$$
\sum_{h=1}^{m}\left((k-k(h)) z_{h}\left(p, \bar{\omega}_{h}\right)+k(h)\left(\left(\bar{z}_{h(\alpha)}\left(p, \bar{\omega}_{h}\right)+\bar{z}_{h(\beta)}\left(p, \bar{\omega}_{h}\right)\right)\right)=0 .\right.
$$

Divide by $k$ and let $\lambda_{h}=k(h) / k$ be the ratio of those $h$-th consumers that are restricted to the total number of $h$-th consumers. Then, the equilibrium equation becomes

$$
\sum_{i=1}^{m}\left(\left(1-\lambda_{h}\right) z_{h}\left(p, \bar{\omega}_{h}\right)+\lambda_{h}\left(\bar{z}_{h(\alpha)}\left(p, \bar{\omega}_{h}\right)+\bar{z}_{h(\beta)}\left(p, \bar{\omega}_{h}\right)\right)=0 .\right.
$$

From now on, the vector of restricted participations $\lambda=\left(\lambda_{1}, \ldots, \lambda_{m}\right)$ is going to vary in the set $\Lambda=[0,1]^{m}$. The value $\lambda=0=(0, \ldots, 0)$ corresponds to the purely Walrasian case, i.e., to full market participation. The value $\lambda=1=(1,1, \ldots, 1)$ corresponds to the polar case of fully restricted market participation.

It will be convenient to consider the open set

$$
\Lambda_{\varepsilon}=\left\{\lambda=\left(\lambda_{1}, \ldots, \lambda_{m}\right) \in \mathbb{R}^{m} \mid-\varepsilon<\lambda_{j}<1+\varepsilon, j=1, \ldots, m\right\}
$$

where $\varepsilon>0$ is usually small. The set $\Lambda_{\varepsilon}$ is useful when dealing with questions involving the boundary of the set $\Lambda$.

We want to make apparent from the notation what parameters are variable for the sake of the analysis in contrast to those that are fixed. The economic model $\mathscr{E}(\lambda)$ consists of the economies defined by $m$ consumers and the associated vector $\lambda$ of market participation where preferences are fixed, but where initial endowments can vary, provided they remain symmetric. The relevant set of initial endowments, or economies, associated with the model $\mathscr{E}(\lambda)$ is, therefore, the set $\bar{\Omega}=\left(\mathbb{R}^{l}\right)^{m}$, where the $\boldsymbol{m}$-tuple of initial endowments $\left(\bar{\omega}_{1}, \bar{\omega}_{2}, \ldots, \bar{\omega}_{m}\right)$ represents the symmetric components of every consumer's initial endowments.

\section{EQUILIBRIUM PRICE VECTORS AND EQUILIBRIUM ALLOCATIONS. REGULAR ECONOMIES}

Equilibrium equation (1) can be rewritten as

$$
z(p, \lambda, \bar{\omega})=z(p, \bar{\omega})+\sum_{h=1}^{n} \lambda_{i}\left(\left(\bar{z}_{h(\alpha)}\left(p, \bar{\omega}_{h}\right)+\bar{z}_{h(\beta)}\left(p, \bar{\omega}_{h}\right)-z_{h}\left(p, \bar{\omega}_{h}\right)\right)=0\right.
$$

where $z(p, \bar{\omega})$ denotes the aggregate excess demand for the purely Walrasian case. Let $E(\lambda, \bar{\omega})$ denote the set of price solutions $p=(p(\alpha), p(\beta)) \in S$ to equation (2). The set $E(\lambda, \bar{\omega})$ consists of the equilibrium price vectors associated with the vector of restricted participation $\lambda \in \Lambda$ and the initial endowments $\bar{\omega} \in \bar{\Omega}$. The properties of equilibria, including the sunspot equilibria, as a function of market participation are all embodied in the equilibrium correspondence $\lambda \rightarrow \mathrm{E}(\lambda, \bar{\omega})$, with $\bar{\omega}$ fixed. Because of the lack of any normalization for the price vector $p=(p(\alpha), p(\beta)) \in S$, it follows that, if $p$ is an equilibrium price vector associated with the pair $(\lambda, \bar{w})$, then any scalar multiple $\mu p$, with $\mu>0$, is also an equilibrium price vector. This means that non-normalized equilibrium price vectors are never isolated. A standard way to cope with this problem when one wants to define regular 
equilibria is to use normalization assumptions. An alternative, but nevertheless equivalent route, is to consider the set of equilibrium allocations. Let $A(\lambda, \bar{\omega})$ denote therefore the set of equilibrium allocations

$$
x=\left(x_{h}\right)_{h \in H} \in\left(\mathbb{R}^{2 l} \times \mathbb{R}^{2 l}\right)^{m}
$$

associated with the economy parametrized by $\lambda \in \Lambda$ and $\bar{\omega} \in \bar{\Omega}$. The equation system that $x$ must satisfy in order to be an equilibrium allocation of the economy $(\lambda, \bar{\omega})$ is obtained by writing down the first-order conditions that each $x_{h}$ must satisfy plus the total resource constraint. (We will see explicit computations displaying these equations later.) This equation system is equivalent to the equation system (2) in the sense that any solution of (2) yields an equilibrium allocation, and conversely. Furthermore, the transformations that enable one to go from one of these equation systems to the other can be shown to be smooth. Therefore, this enables one to define regularity directly in terms of the equation system defining $A(\lambda, \bar{\omega})$. Though this is equivalent to the standard approach utilizing aggregate excess demand and normalized prices, it will turn out later that the direct study of the equation system defining $A(\lambda, \bar{\omega})$ will lend itself to simpler, more direct, computations in dealing with the regularity of equilibria in the sunspot model.

At this stage, the following notation is introduced. When $\lambda$ is fixed, the set of regular symmetric economies $\mathscr{R}(\lambda) \subset \bar{\Omega}$ is defined as the set of regular values of the parameter $\bar{\omega} \in \bar{\Omega}$. (Recall the basic intuition that $\bar{\omega}$ is a regular value if the equilibrium equation has no root with multiplicity higher than one, i.e., no double, triple, etc., roots.) The set of singular (symmetric) economies $\sum(\lambda)$ is the complement of $\mathscr{R}(\lambda)$ in $\bar{\Omega}$.

\section{The certainty economy}

The model $\mathscr{E}(\lambda)$ is closely related to a reduced-form model that we define as follows and that does not depend on the parameter $\lambda$. There are $l$ commodities, $m$ Walrasian consumers defined by the utility functions $v_{h}$ and initial endowments $\bar{\omega}_{h}$ for $h=1,2, \ldots, m$. Let $\bar{E}$ denote the model defined by these economies, $\bar{x}$ and $\bar{\Sigma}$ their set of regular and singular economies respectively. Let $\bar{z}(\bar{p}, \bar{\omega})=\sum_{h=1}^{m} \tilde{z}_{h}\left(\bar{p}, \bar{\omega}_{h}\right)$ be the aggregate excess demand of the certainty economy. Let $\bar{E}(\omega)$ denote the set of equilibrium price vectors associated with a given $\bar{\omega} \in \bar{\Omega}$, and $A(\omega)$ the corresponding set of equilibrium allocations.

\section{The non-existence of sunspot equilibria for unrestricted participation}

The following lemma recalls an important property of the purely Walrasian case, namely, the lack of sunspot equilibria when there are no restrictions on market participation.

Lemma 3.1. The equilibrium allocation $x=(x(\alpha), x(\beta))$ belongs to $A(0, \bar{\omega})$ if and only if $x$ is symmetric, i.e., $x(a)=x(\beta)=\bar{x}$, where $\bar{x}$ belongs to the set $A(\bar{\omega})$.

This lemma can be re-phrased with the help of the following formula

$$
A(\mathbf{0}, \bar{\omega})=(\bar{A}(\bar{\omega}) \times \bar{A}(\bar{\omega})) \cap \Delta
$$

where $\Delta$ denotes the diagonal of the Cartesian production $\bar{\Omega} \times \bar{\Omega}$.

Outline of the proof. Given $\lambda=0$, we have a Walrasian economy. Therefore, the first welfare theorem holds true, i.e., every equilibrium allocation $x=(x(\alpha), x(\beta))$ is a Pareto optimum. Now, every Pareto optimum is symmetric. Assume the contrary, i.e., $x \neq x^{\sigma}$ 
where $x^{\sigma}$ is the image of $x$ by the symmetry that exchanges $\alpha$ and $\beta$. Then the allocation $\left(x+x^{\sigma}\right) / 2$ is Pareto superior to $x$, hence a contradiction.

Now, given Lemma 1 , we have $z_{h}\left(\pi(\alpha) \bar{p}, \pi(\beta) \bar{p}, \bar{\omega}_{h}\right)=\left(\bar{z}_{h}\left(\bar{p}, \bar{\omega}_{h}, \bar{z}_{h}\left(\bar{p}, \bar{\omega}_{h}\right)\right)\right.$. Therefore, the equilibrium equation for the purely Walrasian economy,

$$
\sum_{h=1}^{m} z_{h}\left(\pi(\alpha) \bar{p}, \pi(\beta) \bar{p}, \bar{\omega}_{h}\right)=0,
$$

is equivalent to

$$
\sum_{h=1}^{n} \bar{z}_{h}\left(\bar{p}, \bar{\omega}_{h}\right)=0,
$$

which is the equilibrium equation of the certainty economy, i.e., $\bar{p} \in \bar{E}(\bar{\omega})$, and therefore $\bar{x}$ belongs to $\bar{A}(\bar{\omega})$.

Lemma 3.2. The set $A(1, \bar{\omega})$ is equal to the Cartesian product $\bar{A}(\bar{\omega}) \times \bar{A}(\bar{\omega})$.

Proof. The equilibrium equation defining the equilibrium price vector $p \in E(1, \bar{\omega})$ can be written as

$$
z(p, 1, \bar{\omega})=\sum_{h=1}^{m}\left(z_{h(\alpha)}\left(p, \bar{\omega}_{h}\right)+z_{h(\beta)}\left(p, \bar{\omega}_{h}\right)\right)=\mathbf{0} \in \mathbb{R}^{2 l}
$$

which is equivalent to

$$
z(p, 1, \bar{\omega})=\left(\left(\sum_{h=1}^{m} \bar{z}_{h}\left(p(\alpha), \bar{\omega}_{h}\right)\right)\left(\sum_{h=1}^{m} \bar{z}_{h}\left(p(\beta), \bar{\omega}_{h}\right)\right)\right)=(\mathbf{0}, \mathbf{0}) \in \mathbb{R}^{\prime} \times \mathbb{R}^{l}
$$

and, therefore, to having $\bar{z}(p(\alpha), \bar{\omega}))=\bar{z}(p(\beta), \bar{\omega}))=0$, i.e., to $p(\alpha)$ and $p(\beta) \in \bar{E}(\bar{\omega})$. This implies that the corresponding allocations $x(\alpha)$ and $x(\beta)$ both belong to $\bar{A}(\bar{\omega})$. The converse is obvious. $\|$

This result, first observed by Cass and Shell (1983), readily implies that if $n$ denotes the number of elements of $\bar{A}(\bar{\omega})$, then the Cartesian product $\bar{A}(\bar{\omega}) \times \bar{A}(\bar{\omega})$ contains $n^{2}$ elements, only $n$ of which are symmetric, which implies that there exist $n^{2}-n$ sunspot equilibria. Therefore, one can expect from these two lemmas that, when $\lambda$ varies between the two polar cases defined by $\lambda=0$ and $\lambda=1$, the equilibrium set is likely to vary as a function of $\lambda$ in order to change from a set containing only $n$ elements into another one containing $n^{2}$ elements. (This analysis makes sense only if $n$ is strictly larger than one.) The next lemma shows that, incidentally, the set of non-sunspot equilibrium allocations does not depend on the parameter $\lambda$ :

\section{Lemma 3.3. We have}

$$
A(\mathbf{0}, \bar{\omega})=A(\lambda, \bar{\omega}) \cap \Delta \text { for every } \lambda \in \Lambda .
$$

Proof. Let $p=(\bar{p}, \bar{p}) \in \Delta$. We have $z_{h}\left(p, \bar{\omega}_{h}\right)=\left(\bar{z}_{h}\left(\bar{p}, \bar{\omega}_{h}\right), \bar{z}_{h}\left(\bar{p}, \bar{\omega}_{h}\right)\right), z_{h(\alpha)}\left(p, \bar{\omega}_{h}\right)=$ $\left(\bar{z}_{h}\left(\bar{p}, \bar{\omega}_{h}\right), 0\right)$ and $\left.z_{h(\beta)}\right)\left(p, \bar{\omega}_{h}\right)=\left(0, \bar{z}_{h}\left(\bar{p}, \bar{\omega}_{h}\right)\right)$. Thus, equation (2) becomes

$$
z(p, \lambda, \bar{\omega})=z(p, \bar{\omega})+\sum_{h=1}^{m} \lambda_{h}\left(\left(\bar{z}_{h}\left(\bar{p}, \bar{\omega}_{h}\right), 0\right)+\left(0, \bar{z}_{h}\left(\bar{p}, \bar{\omega}_{h}\right)\right)-\left(\bar{z}_{h}\left(\bar{p}, \bar{\omega}_{h}\right), \bar{z}_{h}\left(\bar{p}, \bar{\omega}_{h}\right)\right)\right)=0
$$

so

$$
z(p, \lambda, \bar{\omega})=z(p, \bar{\omega})=0
$$

for every $\lambda \in \Lambda$. Therefore, whenever $p$ is symmetric, $z(p, \lambda, \bar{\omega})=0$ is equivalent to $z(p, \bar{\omega})=$ 0 , i.e., $E(\lambda, \bar{\omega}) \cap \Delta=E(0, \bar{\omega})$. $\|$ 
It follows from Lemmas 2 to 4 that, when $\lambda$ varies between the two polar cases defined by $\lambda=\mathbf{0} \in \mathbf{\Lambda}$ and $\lambda=\mathbf{1} \in \mathbf{\Lambda}$, changes in the set of equilibrium allocations $A(\lambda, \vec{\omega})$ (particularly the number of its elements) involve only the sunspot equilibria, not the nonsunspot equilibria. A natural question to ask is whether the properties stated in Lemmas 2 and 3 are robust to perturbations, and, therefore, whether they remain true for $\lambda$ varying in neighbourhoods of $\mathbf{0}$ and 1 respectively.

The non-existence of sunspot equilibria when the restrictions on market participation are small enough

The following theorem describes how the equilibria of an economy are affected when only a small fraction of the agents are restricted. In fact, there exist no sunspot equilibria for such economies, a property which can also be understood as a statement about the robustness of the non-existence of sunspot equilibria and about the extent to which the economy can absorb agents facing restrictions in market participation without the overall equilibrium outcome being affected at all.

Theorem 2.4. Let $\overline{\boldsymbol{\omega}} \in \overline{\mathscr{R}}$ be a regular economy (of the certainty model $\bar{\S}$ ). There exists an open neighbourhood $V$ of $0=(0,0, \ldots, 0)$ in $\Lambda_{\varepsilon}$ such that, for $\lambda \in V$, we have $A(\lambda, \bar{\omega})=$ $A(\mathbf{0}, \tilde{\omega})$.

Proof. This theorem is a statement about the shape of the graph of the equilibrium correspondence $\lambda \rightarrow A(\lambda, \bar{\omega})$ in a neighbourhood of the origin $0=(0, \ldots, 0)$ for fixed $\bar{\omega}$. We already known from Lemma 2 that $A(\lambda, \bar{\omega})$ contains $A(\mathbf{O}, \bar{\omega})$ for any $\lambda \in \Lambda$. Therefore, all we have to prove is that the graph of $\lambda \rightarrow A(\lambda, \bar{\omega})$ contains in a neighbourhood of $0=$ $(0, \ldots, 0)$ no other branches except for the constant branches that originate from the elements of $A(0, \bar{\omega})$. With the notation used in Appendix $A$, consider the equilibrium set $A=\left\{(x, \lambda, \omega) \in \Omega \times\left(\Lambda_{\varepsilon} \times \Omega\right) \mid x\right.$ is an equilibrium allocation associated with $\lambda$ and $\left.\omega\right\}$ and the projection $\pi: A \rightarrow \Lambda_{\varepsilon} \times \Omega$ where, by definition, $\Omega=\bar{\Omega} \times \bar{\Omega}$ represents not necessarily symmetric endowments.

The property stated in the theorem will be proved if we can show that the mapping $\pi: A \rightarrow \Lambda_{\varepsilon} \times \Omega$ defines a finite covering in a neighbourhood of $\{0\} \times \omega$ where $\omega=(\bar{\omega}, \bar{\omega})$ (see, e.g., Balasko $(1988,4.2 .3)$ ). Since $A$ is a smooth manifold and $\pi$ a proper mapping (cf., Appendix A), it suffices to show that $(0, \omega)$ is a regular economy, which is equivalent to showing that no equilibrium $(x, 0, \omega)$ is critical, i.e., that $\omega=(\bar{\omega}, \bar{\omega})$ is a regular economy or, equivalently, that $\bar{\omega}$ belongs to the subset $\mathscr{R}(0)$ of regular (symmetric) economies in $\overline{\mathbf{\Omega}}$. $\|$

Lemma 3.5. The sets of regular economies $\mathscr{R}(0)$ and $\overline{\mathscr{R}}$ are identical.

Proof. The idea of the proof is to show that the equilibrium $((\bar{x}, \bar{x}), \mathbf{0},(\bar{\omega}, \bar{\omega}))$ is regular if and only if the equilibrium $(\bar{x}, \bar{\omega})$ of the certainty economy is itself regular. Regularity means that suitably defined determinants (for the sunspot economy and for the certainty economy, respectively) are not equal to zero. The proof will then follow from the observation that these determinants are equal to zero simultaneously. An equilibrium of the sunspot economy satisfies the following equation system, where the unknowns are the allocations $x(\alpha)$ and $x(\beta)$, and the Lagrange multipliers $\mu_{2}, \ldots, \mu_{m}$ associated with 
the utility constraints (used in the definition of a Pareto optimum):

$$
\begin{gathered}
\\
\left\{\begin{array}{l}
\sum_{h} x_{h}(\alpha)-\bar{r}=0 \\
\mu_{2} D v_{2}\left(x_{2}(\alpha)\right)-D v_{1}\left(x_{1}(\alpha)\right)=0 \\
\vdots \\
\mu_{m} D v_{m}\left(x_{m}(\alpha)\right)-D v_{1}\left(x_{1}(\alpha)\right)=0
\end{array}\right. \\
\left\{\begin{array}{l}
\sum_{h} x_{h}(\beta)-\bar{r}=0 \\
\mu_{2} D v_{2}\left(x_{2}(\beta)\right)-D v_{1}\left(x_{1}(\beta)\right)=0 \\
\vdots \\
\mu_{m} D v_{m}\left(x_{m}(\beta)\right)-D v_{1}\left(x_{1}(\beta)\right)=0
\end{array}\right. \\
\left\{\begin{array}{c}
\pi(\alpha) D v_{2}\left(x_{2}(\alpha)\right)\left(x_{2}(\alpha)-\bar{\omega}_{2}\right)+\pi(\beta) D v_{2}\left(x_{2}(\beta)\right)\left(x_{2}(\beta)-\bar{\omega}_{2}\right)=0 \\
\vdots
\end{array}\right.
\end{gathered}
$$

The Jacobian matrix associated with these equations taken with respect to $x_{1}(\alpha), \ldots$, $x_{m m}(\alpha), x_{1}(\beta), \ldots x_{m}(\beta)$ and $\mu_{2}, \ldots, \mu_{m}$ is made up of three parts that reflect the structure of the equation system.

$$
\left[\begin{array}{ccc}
A(x(\alpha)) & 0 & D(x(\alpha)) \\
0 & A(x(\beta)) & D(x(\beta)) \\
B(x(\alpha)) & B(x(\beta)) & 0
\end{array}\right]=\mathscr{A}(x(\alpha), x(\beta)) .
$$

At a non-sunspot equilibrium, we have $x(\alpha)=x(\beta)=\bar{x}$. It follows that $A(x(\alpha))=$ $A(x(\beta))=\bar{A}$, that $B(x(\alpha))=B(x(\beta))=\bar{B}$, and that $D(x(\alpha))=D(x(\beta))=\bar{D}$. The Jacobian matrix is therefore equal to

$$
\mathscr{A}(\ddot{x}, \bar{x})=\left[\begin{array}{ccc}
\bar{A} & 0 & \bar{D} \\
0 & \bar{A} & \bar{D} \\
\bar{B} & \bar{B} & 0
\end{array}\right] .
$$

Similarly, the conditions satisfied by an equilibrium of the certainty economy take the form

$$
\begin{aligned}
& \left\{\begin{array}{c}
\sum_{h} \bar{x}_{h}-\bar{r}_{2}=0 \\
\mu_{2} D v_{2}\left(\bar{x}_{2}\right)-D v_{1}\left(\bar{x}_{1}\right)=0 \\
\vdots \\
\mu_{m} D v_{m}\left(\bar{x}_{m}\right)-D v_{1}\left(\bar{x}_{1}\right)=0
\end{array}\right. \\
& \left\{\begin{array}{c}
D v_{2}\left(\bar{x}_{2}\right)\left(\bar{x}_{2}-\bar{\omega}_{2}\right)=0 \\
\vdots \\
D v_{m}\left(\bar{x}_{m}\right)\left(\bar{x}_{m}-\bar{\omega}_{m}\right)=0 .
\end{array}\right.
\end{aligned}
$$

Straightforward computations of the Jacobian matrix with respect to $\bar{x}$ and $\mu_{2}, \ldots, \mu_{m}$ show that this matrix is equal to

$$
\left[\begin{array}{ll}
\bar{A} & \bar{D} \\
\bar{B} & (0)
\end{array}\right]=\bar{A}(\bar{x})
$$


where $\bar{A}, \bar{B}$ and $\bar{D}$ have been defined previously. It is clear that regularity of $(\bar{x}, \bar{x}),(0)$, $(\bar{\omega}, \bar{\omega}))$ is equivalent to det $\mathscr{A}(\bar{x}, \bar{x}) \neq 0$ while regularity of $(\bar{x}, \bar{\omega})$ is equivalent to det $\overline{\mathscr{A}}(x) \neq 0$. It suffices to establish that det $\mathscr{A}(x, \bar{x})=0$ if and only if det $\overline{\mathscr{A}}(\bar{x})=0$.

Assume $\operatorname{det} \mathscr{A}(\bar{x}, \bar{x})=0$. The row vectors of $\mathscr{A}(\bar{x}, \bar{x})$ are linearly dependent: let $a_{k_{\alpha}}, a_{k_{\beta}}$ and $b_{k}^{\prime}$ be the coefficients associated with the rows $k_{\alpha}, k_{\beta}$ and $k^{\prime}$ in some linear dependence relation, respectively. Let $r_{k_{a}}, r_{k_{\beta}}$ and $r_{k^{\prime}}$ denote these row vectors. By definition, we have

$$
\sum a_{k_{a}} r_{k_{a}}+\sum a_{k_{\beta}} r_{k_{\beta}}+\sum b_{k^{\prime}} r_{k^{\prime}}=0
$$

Let $\bar{r}_{k}, \bar{r}_{k}$ denote the row vectors of the matrix $\overline{\mathscr{A}}(\bar{x})$. The above linear dependence relation implies that the coefficients $\left(a_{k_{a}}\right)$ and $\left(b_{k^{\prime}}\right)$ define a linear dependence relation for the rows of the matrix

$$
\left(\begin{array}{c}
\bar{A} \\
\pi(\alpha) \bar{B}
\end{array}\right)
$$

while $\left(a_{k_{\beta}}\right)$ and $\left(b_{k^{\prime}}\right)$ define a similar linear dependence relation for the rows of the matrix

$$
\left(\begin{array}{c}
\bar{A} \\
\pi(\beta) \bar{B}
\end{array}\right)
$$

and, similarly, $a_{k_{a}}+a_{k_{\beta}}$ a linear dependence relation for the rows of $\bar{D}$.

It follows from $\pi(\alpha)+\pi(\beta)=1$ that the coefficients defined by the (coordinates of the) vectors $\left(a_{k_{\alpha}}+a_{k_{\beta}}\right)$ and $\left(b_{k^{\prime}}\right)$ define a linear dependence relation for the rows of the matrix

$$
\left(\begin{array}{cc}
\bar{A} & \bar{D} \\
\bar{B} & 0
\end{array}\right)=\overline{\mathscr{A}}(\bar{x})
$$

which show det $\overline{\mathscr{A}}(\bar{x})=0$.

Assume now the converse, namely det $\bar{A}(\bar{x})=0$. Let $\left(a_{k}\right)$ and $\left(b_{k^{\prime}}\right)$ be the coefficients of a linear dependence relation between the rows of

$$
\left(\begin{array}{ll}
\bar{A} & \bar{D} \\
\bar{B} & 0
\end{array}\right)
$$

It suffices to take $a_{k_{a}}=\pi(\alpha) \mathrm{a}_{k}$ and $a_{k g}=\pi(\beta) \mathbf{a}_{k}$ to have the coefficients $\left(a_{k_{q}}\right),\left(a_{k_{\beta}}\right)$ and $\left(b_{k^{\prime}}\right)$ define a linear dependence relation between the rows of the matrix

$$
\left(\begin{array}{ccc}
\bar{A} & 0 & \bar{D} \\
0 & \bar{A} & \bar{D} \\
\pi(\alpha) \bar{B} & \pi(\beta) \bar{B} & 0
\end{array}\right),
$$

which implies $\operatorname{det} \mathscr{A}(\bar{x}, \bar{x})=0$.

Since the writing of the first version of this paper, alternative proofs of Lemma 5 using the Jacobian matrix of aggregate demand have been obtained independently by Prechac (1988) and Villanacci (1988). For a more "geometrical" proof, i.e., one that is almost calculation-free, see Appendix B.

We have seen that sunspot equilibria exist for some economies and some level of restrictions on market participation. We have also seen that they do not exist always. 
Therefore, the study of the existence of sunspot equilibria for arbitrarily fixed restrictions on market participation has two facets, at least: the study of economies without sunspot equilibria on the one hand, and the study of the economies with sunspot equilibria on the other. The study of these questions, the existence issue especially, requires us to go through a thorough analysis of the sunspot model for fixed restrictions on market participation. This analysis is carried out in the next section.

\section{A REFORMULATION OF THE MODEL $\mathscr{E}(\lambda)$. ANALYSIS OF THE MODEL OF RESTRICTED MARKET PARTICIPATION}

Let $\lambda \in \Lambda$ be fixed. The study of the model $\mathscr{E}(\lambda)$ goes through a reformulation that yields an equation system related to the equation system of standard general equilibrium theory.

\section{Reformulation of the model $\mathscr{E}(\lambda)$}

We need only to study the model $\mathscr{E}(\lambda)$ for values of $\lambda=\left(\lambda_{1}, \ldots, \lambda_{m}\right)$ that are $m$-tuples of rational numbers because a straightforward continuity argument enables us to extend to any $\lambda \in \Lambda$ the existence statement proved for $\lambda$ rational.

Therefore, let $\lambda=\left(k_{1} / k, \ldots, k_{m} / k\right)$. The model $\mathscr{E}(\lambda)$ becomes equivalent to the model defined by a finite number of consumers who, besides satisfying the obvious symmetry requirements, are facing either one or two different budget constraints. It turns out to be helpful to introduce the following notation. Let $I$ denote the set of Walrasian, or unconstrained consumers, and $J$ the set of constrained consumers. The polar cases $\lambda=0$ and $\lambda=1$ have been analyzed in Cass-Shell (1983). Therefore we assume from now on that consumer 1 is always unrestricted, i.e., $\{1\} \in I$, which therefore excludes the case $\lambda=1$.

In order to simplify the analysis, we denote by $\mathscr{E}(I, J, \bar{r})$ the economic model consisting of economies defined by initial endowments $\omega=\left(\bar{\omega}_{i}, \bar{\omega}_{i}\right)_{i \in I, j \in J}$ satisfying the total resources requirements, i.e., $\sum_{i \in I} \bar{\omega}_{i+\sum_{j \in \zeta}} \bar{\omega}_{j}=\bar{r}$, the utility functions being defined as in the previous sections (i.e., $u_{h}\left(x_{h}(\alpha), x_{h}(\beta)\right)=\pi(\alpha) v_{h}\left(x_{h}(\alpha)\right)+\pi(\beta) v_{h}\left(x_{h}(\beta)\right)$ for $h \in I \cup J$ ) while the consumer's budget constraints depend on whether he belongs to the set $I$ or to the set $J$.

The certainty model $\overline{\mathscr{E}}(H, \bar{r})$ and the set of Pareto optima $\bar{P}(H, \bar{r})$

Many properties of the model $\mathscr{E}(I, J, \bar{r})$ depend on those of the certainty model $\overline{\mathscr{E}}(H, \bar{r})$ where the set $H=I \cup J$ denotes the set of households, either unrestricted or restricted, the number of commodities in $\overline{\mathscr{E}}(H, \bar{r})$ being $l$, and consumer $h \in \mathbb{R}^{m}$ being characterized by the utility function $v_{h}: \mathbb{R}^{\prime} \rightarrow \mathbb{R}$ and the endowments $\bar{\omega}_{h} \in \mathbb{R}^{\prime}$.

Let $\bar{P}(H, \bar{r})$ denote the set of Pareto optima in the model $\mathscr{E}(H, \bar{r})$. There is an almost obvious relationship between the Pareto optima of the model $\mathscr{E}(I, J, \bar{r})$ (which do not depend on how the set of consumers $H$ decomposes into the subsets $I$ and $J$ ) and the Pareto optima $\bar{P}(H, \bar{r})$ of the model $\mathscr{E}(H, \bar{r})$.

Lemma 4.1. The allocation $x=(x(\alpha), x(\beta))$ is a Pareto optimum of $\mathscr{E}(I, J, \bar{r})$ if and only if:

1. $x(\alpha)=x(\beta)$;

2. The allocation $\bar{x}$ defined by $\bar{x}=x(\alpha)=x(\beta)$ belongs to $\bar{P}(H, \bar{r})$. 
In other words, the set of Pareto optima of $\mathscr{E}(I, J, \bar{r})$ is the diagonal of the Cartesian product $\vec{P}(H, \bar{r}) \times \vec{P}(H, \bar{r})$ and is, therefore, diffeomorphic to $\bar{P}(H, \bar{r})$.

Proof. The first statement of the lemma follows from the observation already made in the proof of Lemma 2 that every Pareto optimal allocation is symmetric. The second statement of the lemma then follows immediately.

It follows from this lemma that the intersection of the set of the Pareto optima of $\mathscr{E}(I, J, \bar{r})$ with the diagonal can be identified with the set of Pareto optima $\bar{P}(H, \bar{r})$. We know that sunspot equilibria can exist only if the first welfare theorem does not hold true, i.e., if the set of equilibrium allocations of the model $\mathscr{E}(I, J, \bar{r})$ is strictly larger than (i.e., in the set-theoretic sense, includes) the set of Pareto optima. The study of the set of equilibrium allocations follows from an alternative interpretation of the model $\mathscr{E}(I, J, \bar{r})$.

The set of equilibrium allocations of the model $\mathscr{E}(I, J, \bar{r})$ and the set of Pareto optima $\bar{P}(H, \bar{r})$

Based on the observation (cf. Section 2) that a consumer $h$ restricted from participating in the suitable markets is formally equivalent to two quasi-Walrasian consumers $h(\alpha)$ and $h(\beta)$, we define the model $\mathscr{F}(I, J(\alpha) \cup J(\beta), \bar{r})$ as follows. It consists of the consumers $h_{u} \in I, h_{r}(\alpha) \in J(\alpha)$ and $h_{r}(\beta) \in J(\beta)$, consumers $h_{r}(\alpha)$ and $h_{r}(\beta)$ now being quasi-Walrasian. This model is mathematically equivalent to $\mathscr{E}(I, J, \vec{r})$ in the sense that it defines the same set of equilibrium equations. There is, however, an important difference between these two models. The first welfare theorem holds true for the model $\mathscr{F}(I, J(\alpha) \cup J(\beta), \bar{r})$, which implies that there is an identity between the equilibrium allocations of $\mathscr{F}(I, J(\alpha) \cup J(\beta), \bar{r})$ (and, for that matter, of $\mathscr{E}(I, J, \bar{r})$ ) and the Pareto optima of $\mathscr{F}(\mathrm{I}, \mathrm{J}(\alpha) \cup \mathrm{J}(\boldsymbol{\beta}), \overline{\mathrm{r}})$. Therefore, the first part of the programme, namely the study of the equilibrium allocations, becomes the simpler one of studying the Pareto optima of the model $\mathscr{F}(I, J(\alpha) \cup J(\beta), \tilde{r})$.

The structure of the set of Pareto optima $\bar{P}(H, \bar{r})$ is well understood (cf. Balasko $(1988,5.2 .4)$ and the discussion at the beginning of Section 5.2). This set is diffeomorphic to $\mathbb{R}^{m-1}$ by being parameterized by the utility levels of all but one agent. It is also possible, when preferences are defined by strictly smooth concave utility functions (here denoted $\left.v_{i}\right)$ to define an alternative diffeomorphism between the set of Pareto optima $\bar{P}(H, \bar{r})$ and $\mathbb{R}^{m-1}$ by considering the set of Lagrange multipliers associated with the constraints $v_{i}\left(x_{i}\right)-\bar{v}_{i}=0$ for $i=2, \ldots, m$ when maximizing $v_{1}\left(x_{1}\right)$. This diffeomorphism is going to play a crucial role in the forthcoming analysis. (See Appendix $\mathrm{C}$ for details.)

The structure of the set of Pareto optima of $\mathscr{F}(I, J(\alpha) \cup J(\beta), \bar{r})$

Let us denote by $P(I, J(\alpha) \cup J(\beta), \bar{r})$ the set of Pareto optima of the model $\mathscr{F}(I, J(\alpha) \cup J(\beta), \bar{r})$. Recall that the element $\{1\}$ belongs to the set $I$.

Let $\Phi_{I}: \bar{P}(H, \bar{r}) \rightarrow \mathbb{R}_{++}^{\# I-1}$ denote the smooth mapping whose value is defined by the Lagrange multipliers associated with the unrestricted consumers $i$ with the exception of consumer 1, i.e., for $i \in I \backslash\{1\}$. Note that $\Phi_{I}$ represents the first $(\# I)-1$ coordinates of the diffeomorphism $\Phi$ defined in Appendix B between the set of Pareto optima and $\mathbb{R}^{m-1}$. Therefore, the preimage by $\Phi_{I}$ of an arbitrary element of $\mathbb{P}_{++}^{\# I-1}$ is diffeomorphic to $\mathbb{R}_{++}^{\# J}$ because it is parametrized by the multipliers $\mu_{j}$ for $j \in J$, as follows from Proposition B.1 in Appendix B. 
We then have the following description of the set of Pareto optima $P(I, J(\alpha) \cup J(\beta), \bar{r})$ and of the supporting price vector associated with such element:

Theorem 4.2. The allocation $x=(x(\alpha), x(\beta))$ belongs to $P(I, J(\alpha) \cup J(\beta), \bar{r})$ if and only if:

1. $x(\alpha)$ and $x(\beta)$ belong to $\vec{P}(H, \bar{r})$;

2. $\Phi_{I}(x(\alpha))=\Phi_{I}(x(\beta))$.

Then, the supporting price vector $(\pi(\alpha) p(\alpha), \pi(\beta) p(\beta))=D u_{1}\left(x_{1}(\alpha), x_{1}(\beta)\right)$ is such that the component $p(\alpha)=D v_{1}\left(x_{1}(\alpha)\right)\left(\right.$ resp. $\left.p(\beta)=D v_{1}\left(x_{1}(\beta)\right)\right)$ supports the Pareto optimum $x(\alpha)$ (resp. $x(\beta)) \in \bar{P}(H, \bar{r})$.

Proof. The allocation $x=(x(\alpha), x(\beta))$ is a Pareto optimum if and only if the firstorder conditions associated with the maximization problem:

$$
\begin{array}{ll}
\text { Maximize } & u_{1}\left(x_{1}(\alpha), x_{1}(\beta)\right) \\
\text { subject to } & \left\{\begin{array}{l}
u_{i}\left(x_{i}(\alpha), x_{i}(\beta)\right)-\bar{u}_{i}=0, \quad j \in I \backslash\{1\}, \\
v_{j}\left(x_{j}(\alpha)\right)-\bar{v}_{j a}=0, \quad j \in J, \\
v_{j}\left(x_{j}(\beta)\right)-\bar{v}_{j \beta}=0, \quad j \in J, \\
\sum_{i \in I} x_{i}+\sum_{j \in J} x_{j}=(\bar{r}, \bar{r}),
\end{array}\right.
\end{array}
$$

are satisfied. Let $\mu_{i}, \mu_{j \alpha}, \mu_{j \beta}$, and $p=(\pi(\alpha) p(\alpha), \pi(\beta) p(\beta))$ be the Lagrange multipliers associated with the respective constraints. The Lagrangean can be written in the following way:

$$
\begin{aligned}
\mathscr{L}\left(x\left(\alpha, x(\beta),\left(\mu_{i}\right)_{i},\left(\mu_{j \alpha}\right)_{j \alpha},\left(\mu_{j \beta}\right)_{j \beta}, \pi(\alpha) p(\alpha), \pi(\beta) p(\beta)\right)=\pi(\alpha) v_{1}\left(x_{1}(\alpha)\right)\right. \\
\left.\quad+\pi(\beta) v_{1}\left(x_{1}(\beta)\right)+\sum_{i \in J \backslash\{1} \mu_{i}\left(\pi(\alpha) v_{i} x_{i}(\alpha)\right)+\pi(\beta) v_{i}\left(x_{i}(\beta)\right)-\bar{\mu}_{i}\right) \\
\quad+\sum_{j \in J}\left(\mu _ { j \alpha } \pi ( \alpha ) \left(v_{j}\left(x_{j}(\alpha)-\bar{v}_{j \alpha}\right)+\mu_{j \beta} \pi(\beta)\left(v_{j}\left(x_{j}(\beta)\right)-\bar{v}_{j \beta}\right)\right.\right. \\
\quad-\pi(\alpha) p(\alpha) \cdot\left(\sum_{h \in I \cup J} x_{h}(\alpha)-\bar{r}\right)-\pi(\beta) p(\beta) \cdot\left(\sum_{h \in / \cup J} x_{h}(\beta)-\bar{r}\right) .
\end{aligned}
$$

The first-order conditions then take the following form where the role of the $\alpha$ and $\beta$ components are highlighted:

$$
\left({ }^{*} \alpha\right)\left\{\begin{array} { l } 
{ D v _ { 1 } ( x _ { 1 } ( \alpha ) ) = p ( \alpha ) } \\
{ \mu _ { i } D v _ { i } ( x _ { i } ( \alpha ) ) = p ( \alpha ) } \\
{ \mu _ { j \alpha } D v _ { j } ( x _ { j } ( \alpha ) ) = p ( \alpha ) } \\
{ \sum _ { h \in J \cup J } x _ { h } ( \alpha ) = \overline { r } }
\end{array} \quad ( { } ^ { * } \beta ) \left\{\begin{array}{l}
D v_{1}\left(x_{1}(\beta)\right)=p(\beta) \\
\mu_{i} D v_{i}\left(x_{i}(\beta)\right)=p(\beta), \\
\mu_{j \beta} D v_{j}\left(x_{j}(\beta)\right)=p(\beta) \\
\sum_{h \in I \cup J} x_{h}(\beta)=\bar{r}
\end{array}\right.\right.
$$

Consider next the problem:

$$
\begin{array}{ll}
\text { Maximize } & v_{1}\left(\bar{x}_{1}\right) \\
\text { subject to } & \left\{\begin{array}{l}
v_{h}\left(\bar{x}_{h}\right)=\bar{v}_{h}, \\
\sum_{h \in I \cup J} \bar{x}_{h}=\bar{r},
\end{array} \quad h \in I \cup J \backslash\{1\},\right.
\end{array}
$$


that characterizes the elements of $\bar{P}(H, \bar{r})$. The first-order conditions become

$$
\text { (*) }^{*}\left\{\begin{array}{l}
D v_{1}\left(\bar{x}_{1}\right)=\bar{p}^{\prime}, \\
\mu_{h}^{\prime} D v_{h}\left(\bar{x}_{h}\right)=\bar{p}^{\prime}, \quad h \in I \cup J \backslash\{1\}, \\
\sum_{h \in I \cup J} \bar{x}_{h}=\bar{r},
\end{array}\right.
$$

They are identical to $\left({ }^{*} \alpha\right)$ (resp. $\left({ }^{*} \beta\right)$ ) for $p^{\prime}=p(\alpha), \mu_{i}^{\prime}=\mu_{i}$, and $\mu_{j}^{\prime}=\mu_{j \alpha}^{\prime}$ (resp. $p^{\prime}=p(\beta), \mu_{i}^{\prime}=\mu_{i}$, and $\left.\mu_{j}^{\prime}=\mu_{j \beta}^{\prime}\right)$ This shows that conditions $\left({ }^{*} \alpha\right)$ and $\left({ }^{*} \beta\right)$ are equivalent to having the respective Lagrange multipliers associated with $x(\alpha)$ and $x(\beta)$ (as Pareto optima of the model $\overline{\mathscr{E}}(H, \bar{r}))$ being equal for the unrestricted consumers. Then, the price vectors $p(\alpha)$ and $\left(p(\beta)\right.$ defined by the relationships $p(\alpha)=D v_{1}\left(x_{1}(\alpha)\right)$ and $p(\beta)=$ $D v_{1}\left(x_{1}(\beta)\right)$ are such that the price vector $p=(\pi(\alpha) p(\alpha), \pi(\beta) p(\beta))$ supports $(x(\alpha), x(\beta))$. \|

\section{EXISTENCE OF SUNSPOT EQUILIBRIA}

Our goal in this section is to establish two properties regarding the existence of sunspot equilibria. The first result is that if $\bar{\omega} \in \bar{\Omega}$ is sufficiently close to the set of Pareto optima $\bar{P}(H, \bar{r})$ or, equivalently, if $\omega=(\bar{\omega}, \bar{\omega})$ is sufficiently close to the set of Pareto optima of the model $\mathscr{E}(I, J, \bar{r})$, i.e., the set of Pareto optima defined for the set of households $H=I \cup J$, households defined on $\left(\mathbb{R}^{\prime}\right) \times\left(\mathbb{B}^{\prime}\right)$ by the utility function $u_{h}\left(x_{h}(\alpha), x_{h}(\beta)\right)=$ $\left.\pi(\alpha) v_{h}\left(x_{h}(\alpha)\right)+\pi(\beta) v_{h}(\beta)\right)$, then no sunspot equilibrium exists. The second result provides sufficient conditions for the existence of sunspot equilibria for suitably chosen economies $\overline{\boldsymbol{\omega}} \in \overline{\boldsymbol{\Omega}}$.

The non-existence of sunspot equilibria when $\bar{\omega} \in \bar{\Omega}$ is close to the set of Pareto optima $\bar{P}(H, \bar{r})$

Let us consider the model $\mathscr{\mathscr { \sigma }}(I, J, \bar{r})$. Recall that initial endowments $\omega=(\bar{\omega}, \bar{\omega})$ are symmetric. Therefore saying that $\bar{\omega}$ is close to $\bar{P}(H, \bar{r})$ is the same thing as saying that $\omega=(\bar{\omega}, \bar{\omega})$ is close to the set of Pareto optima of $\mathscr{E}(I, J, \bar{r})$. We then have:

Theorem 5.1. There exists an open neighbourhood $U$ of $\bar{P}(H, \bar{r})$ in $\bar{\Omega}$ such that for any $\overline{\boldsymbol{\omega}} \in U$ there are no sunspot equilibria.

Proof. Allow for the moment symmetric endowments that are not necessarily symmetric provided that total resources are symmetric and fixed. We can readily apply to this framework the standard results of general equilibrium theory. Then, there exists an open dense set of regular economies (endowments). Furthermore an open connected component $V$ of this set contains the set of Pareto optima and the equilibrium is unique for $\omega$ in this component $V$. The intersection of the open set $V$ with the diagonal $\Delta$ of $\bar{\Omega} \times \bar{\Omega}$ can be written as $V \cup \Delta=U \times U$. The set $U$ is open, nonempty and contains $\bar{P}(M, \bar{r})$. Now, the set of equilibrium allocations for the certainty model $\mathscr{E}(I, J, \bar{r})$ is non-empty for any $\bar{\omega} \in \bar{\Omega}$. Furthermore, the equilibrium is unique for $\bar{\omega} \in U$. Therefore, there can exist only one nonsunspot equilibrium and no sunspot equilibria for $\bar{\omega} \in U$.

Theorem 5.1 is actually a special case of a similar property satisfied in the more general setup of equivariant general equilibrium theory; see Balasko (1990). 


\section{Symmetric initial endowments compatible with an equilibrium allocation}

We have studied in Section 4 the set of equilibrium allocations of the model $\mathscr{E}(I, J, \bar{r})$. This set can be identified with the set of Pareto optima $P(I, J(\alpha) \cup J(\beta), \bar{r})$. It is quite obvious that every such allocation is the equilibrium allocation of at least one economy. (It suffices to take as an example of an economy the allocation itself, i.e., to take $\omega$ equal to $x$.) The difficulty, however, is that we are only interested in the equilibrium allocations that are associated with symmetric endowments and, in particular, in the asymmetric allocations that are associated with symmetric endowments. Then, it is not at all clear that an arbitrarily chosen element of $P(I, J(\alpha) \cup J(\beta), \bar{r})$ can be an asymmetric equilibrium associated with some symmetric endowment (or economy). Our next result characterizes the allocations that are compatible with symmetric economies. Let $x=$ $(x(\alpha), x(\beta)) \in P(I, J(\alpha) \cup J(\beta), \bar{r})$ and let $p(\alpha)=D v_{1}\left(x_{1}(\alpha)\right)$ and $p(\beta)=D v_{1}\left(x_{1}(\beta)\right)$ be the supporting price vectors for $x(\alpha)$ and $x(\beta)$. Recall that $(\pi(\alpha) p(\alpha), \pi(\beta) p(\beta))$ then supports the allocation $x \in \mathbb{R}^{2 l}$. We have:

Theorem 5.2. There exists a symmetric endowment $\omega=(\bar{\omega}, \bar{\omega})$ compatible with the equilibrium allocation $x=(x(\alpha), x(\beta))$ if and only if the linear equation system

$$
\left\{\begin{array}{l}
p(\alpha) \cdot \bar{\omega}_{j}=p(\alpha) \cdot x_{j}(\alpha), \\
p(\beta) \cdot \bar{\omega}_{j}=p(\beta) \cdot x_{j}(\beta), \quad j \in J,
\end{array}\right.
$$

has a solution for every $j \in J$.

Proof. Let $\left.\bar{\omega}=\left(\bar{\omega}_{i}\right),\left(\bar{\omega}_{j}\right)\right)_{i \in I, j \in J}$ represent the unknown symmetric component. It has to satisfy the following linear equations that represent resource and budget constraints:

(i) $\sum_{i \in I} \bar{\omega}+\sum_{j \in J} \bar{\omega}_{j}=\overline{\mathrm{r}}$,

(ii) $\quad(\pi(\alpha) p(\alpha)+\pi(\beta) p(\beta)) \cdot \bar{\omega}_{i}=\pi(\alpha) p(\alpha) \cdot x_{i}(\alpha)+\pi(\beta) p(\beta) \cdot x_{i}(\beta), \quad i \in I$,

(iii $\alpha) p(\alpha) \cdot \bar{\omega}_{j}=p(\alpha) \cdot x_{j}(\alpha), \quad j \in J$,

(iii $\beta) p(\beta) \cdot \bar{\omega}_{j}=p(\beta) \cdot x_{j}(\beta)$,

This system of linear equations has a solution if and only if equations (iii $\alpha$ ) and (iii $\beta$ ) can be solved for every $j \in J$. If (iii $\alpha$ ) ard (iii $\beta$ ) have no solutions for at least one $j \in J$, there clearly cannot be an $\bar{\omega}$ compatible with $x=(x(\alpha), x(\beta))$. On the other hand, let $\bar{\omega}_{j}$ denote a solution to (iii $\alpha$ ) and (iii $\beta$ ) for every $j \in J$. Obviously, one can then find solutions $\bar{\omega}_{i}$ to (ii) for every $i \in I \backslash\{1\}$. It then suffices to compute $\bar{\omega}_{1}$ by equation (i). The solution $\bar{\omega}_{1}$ then satisfies equation (ii); this follows from adding together the inner product by $\pi(\alpha) p(\alpha)$ of $\sum_{h \in I \cup J} x_{h}(\alpha)=\bar{r}$ and by $\pi(\beta) p(\beta)$ of $\sum_{h \in J \cup J} x_{h}(\beta)=\bar{r}$, respectively, combined with (ii), (iii $\alpha$ ) and (iii $\beta$ ) for all $i \in I \backslash\{1\}$ and all $j \in J$. \|

Corollary 5.3. A sufficient condition for the existence of symmetric endowments $\omega=$ $(\bar{\omega}, \bar{\omega})$ compatible with the equilibrium allocation $x=(x(\alpha), x(\beta))$, where $x(\alpha) \neq x(\beta)$, is

$$
\operatorname{rank}\left(D v_{1}\left(x_{1}(\alpha)\right), D v_{1}\left(x_{1}(\beta)\right)\right)=2 .
$$

\section{Proof. Obvious. \|}

Theorem 5.2 and its corollary reduce the analysis of economies having sunspot equilibria into a problem that can be studied by looking at the simpler certainty model $\overline{\mathscr{E}}(H, \bar{r})$, which consists of $l$ commodities, total resources $\bar{r}$ and consumer $h$ (with $h \in H=I \cup J$ ) defined by the utility function $v_{h}: \mathbb{R}^{\prime} \rightarrow \mathbb{R}$. 


\section{Existence of sunspot equilibria}

Consider the certainty model $\mathscr{E}(H, \bar{r})$. Define in $\bar{\Omega}$ the subset

$$
\bar{\Omega}_{\geqq 2}=\{\bar{\omega} \in \bar{\Omega} \mid \text { there exist at least two equilibria associated with } \bar{\omega}\}
$$

of economies with multiple equilibria. We consider only the models for which this set $\bar{\Omega}_{\geqq 2}$ is non-empty. (Recall that, from Balasko (1988, 7.3.9), models where the equilibrium price vector $\bar{p}$ associated with $\bar{\omega} \in \bar{\Omega}$ is unique are such that $\bar{p}$ is in fact constant, which also means that the supporting price vector of every Pareto optimum $\bar{x} \in \bar{P}(H, \bar{r})$ is then the constant price vector $\bar{p}$; it is not difficult to check that the complement of the set generated by these models is open dense.)

We then have the following result on the existence of the sunspot equilibria:

Theorem 5.4. Assume $\bar{\Omega}_{\geq 2} \neq \varnothing$ (i.e., there exists at least one economy $\bar{\omega}$ with multiple equilibria). There exists at least one consumer $j \in H, j \neq 1$, such that restricting him implies that the subset of $\bar{\Omega}$ consisting of economies with sunspot equilibria is non-empty.

Proof. It follows from Corollary 5.3 to Theorem 5.2 that the existence of sunspot equilibria depends on the variation of the supporting price vector associated with every Pareto optimum when the welfare weights are varied. Using an arbitrary normalization convention (e.g., the numeraire assumption), existence of sunspot equilibria then depends on whether the supporting price vector is constant, or not. It is constant when the welfare weights are varied if and only if there exists no economy $\bar{\omega}$ with multiple equilibria. On the other hand, if $\bar{\Omega}_{\geqq 2}$ is $\neq \varnothing$, this means that there exists at least one combination of the welfare weights $\mu=\left(\mu_{2}^{*}, \ldots, \mu_{m}^{*}\right)$ such that the associated supporting price vector is not locally constant. Then there necessarily exists a pair of multipliers $\mu_{j}$ and $\mu_{j}^{\prime}$ with $\mu_{j} \neq \mu_{j}^{\prime}$ such that the price vectors supporting the Pareto optima associated with $\left.\mu_{2}^{*}, \ldots, \mu_{j-1}^{*}, \mu_{j}, \mu_{j+1}^{*}, \ldots, \mu_{m}^{*}\right)$ and $\left(\mu_{2}^{*}, \ldots, \mu_{j-1}^{*}, \mu_{j}^{\prime}, \mu_{j+1}^{*}, \ldots, \mu_{m}^{*}\right)$ are different. This property is sufficient to imply the existence of sunspot equilibria for any set $J$ of restricted households that contains the element $j$.

The proof of Theorem 5.4 highlights the importance of those consumers that have the property that changing their welfare weights induces a change in the supporting price vector of the associated Pareto optimum. Define these consumers to be price-potent consumers. These consumers exist if and only if the price vector that supports the Pareto optima is not constant, total resources being kept fixed. This property is equivalent to the existence in the certainty model of endowments (sign restrictions being excluded) for which there exist multiple equilibria. The latter property is known to be satisfied for a generic set of preferences.

We have therefore shown in this paper that restricting market participation of the price-potent consumers that exist generically creates sunspot equilibria for suitably chosen endowments.

\section{APPENDICES}

\section{A. WALRASIAN AND QUASI-WALRASIAN CONSUMERS}

In this appendix, we recall the setup of economies where types of agents are fixed; we also recall the properties of these models that are useful in the main text of this paper. Here, the notation departs at places from that in the main text in order to be consistent with that of Balsko (1988). 
The equilibrium model with Walrasian consumers

A consumer of type $i$ is defined by his smooth preferences and initial endowments $\omega_{i}$. We assume that every standard hypothesis is satisfied (cf., for example, Balsko (1988)), so that we can represent his demand given the price vector $p$ by $f_{i}\left(p, p \cdot \omega_{i}\right)$, and his excess demand by $f_{i}\left(p, p \cdot \omega_{i}\right)-\omega_{i}$. Assume that $n_{i}$ consumers of type $i$ exist. Then, the equilibrium equation becomes

$$
\sum_{y=1}^{m} n_{i}\left(f_{i}(p, p \cdot \omega)-\omega_{i}\right)=0
$$

The solutions of this equation do not depend on having the equation multiplied by some arbitrary factor. For example, one could multiply it by $1 / \sum n_{i}$ and, therefore, the equation would become equivalent to

$$
z(p, \lambda, \omega)=\sum_{i=1}^{m} \lambda_{i}\left(f_{i}\left(p, p \cdot \omega_{i}\right)-\omega_{i}\right)=0
$$

where $\lambda_{i}=n_{i} / \sum n_{i}$ then represents the ratio of the number of agents of type $i$ to the total number of consumers. Though the $\lambda_{i}$ should, in principle, be rational numbers, there is no mathematical difficulty in considering a more general model where $\lambda=\left(\lambda_{i}\right)$ would simply belong to the $n$-simplex (i.e., $\lambda_{i} \geqq 0$, for $i=1, \ldots, m$ and $\sum \lambda_{i}=1$ ) without the coordinates $\lambda_{i}$ necessarily being rational numbers. (Note that, depending on the context, there may exist more natural ways of weighting the various types of agents. Such is the case in the third section of the main text of the paper where weights are defined with respect to triples of consumers).

Therefore, in order to cover the variety of all possible settings, we consider a set of parameters $\boldsymbol{\Lambda}$ which is convex and relatively open as a convex subset of $\mathbb{R}^{m}$. Note that closed convex sets can always be embedded into relatively open larger convex sets, so that the above assumption is sufficient for most practical purposes.

We define an economy by the vector parameter $(\lambda, \omega)=\left(\lambda_{1}, \ldots, \lambda_{m}, \omega_{1}, \ldots, \omega_{m}\right) \in \mathbf{\Lambda} \times \Omega$ where $\left.\Omega=\mathbb{R}^{\prime}\right)^{m}$ denotes the space of initial endowments. Let $S$ be the set of price vectors. We define the equilibrium set

$$
E=\{(p, \lambda, \omega) \in S \times \Lambda \times \Omega \mid p \in S \text { is an equilibrium price vector associated with }(\lambda, \omega)\}
$$

and

$$
\pi: E \rightarrow \Lambda \times \Omega
$$

the natural projection. We have:

Theorem A.1. The equilibrium set is a smooth sub-manifold embedded in $S \times \Lambda \times \Omega$. The natural projection $\pi: \boldsymbol{E} \rightarrow \mathbf{\Lambda} \times \mathbf{\Omega}$ is proper.

Proof. The proof parallels the one for the set up of the general equilibrium model; adding the extra parameter $\lambda \in \Lambda$ changes nothing in the analysis. (cf., Balasko (1988). Chapter IV.) \|

Remark. It should be clear from the argument that some assumptions about the parameter set $\Lambda$ could easily be relaxed if necessary.

\section{Equilibrium and Pareto optima with Walrasian and quasi-Walrasian consumers.}

We now consider quais-Walrasian consumers. Define for consumer $i$ the set Supp(i) of commodities that constitute his consumption space where $L=\{1,2, \ldots, I\}$ is the set of commodities. The equality $\operatorname{Supp}(i)=L \operatorname{means}$ that consumer $i$ is Walrasian. We now define the following property of the model $\mathscr{E}(\lambda)$ associated with $\lambda$ (for variable $\omega$ ). We say that the model is indecomposable if the aggregate excess demand function $p \rightarrow z(p, \lambda, \omega)$ cannot be decomposed in the following sense: it is not possible to find a partition of the commodity set into two subsets such that the components of the aggregate excess demand associated with this partition $z(p, \lambda, \omega)=$ $\left(z^{1}(p, \lambda, \omega), x^{2}(p, \lambda, \omega)\right.$ are such that $z^{1}$ (resp. $z^{2}$ ) depends only on the component $p^{1}$ of the price vector (resp. $p^{2}$ ) where $p=\left(p^{1}, p^{2}\right)$. Clearly, the decomposability property crucially depends on whether there exist enough agents whose consumption spaces overlap so that their union is the whole commodity space. Note that the indecomposability property is trivially satisfied whenever there exists at least one (or a positively weighted) Walrasian consumer.

Let us define the subset $\Lambda$, of $\Lambda$ by

$$
\Lambda_{r}=\{\lambda \in \Lambda \text { such that the model is indecomposable }\} .
$$

Then, the following extension of Theorem A.1 is true.

Theorem A.2. The set of equilibria $(p, \lambda, \omega)$ over $\left.\Lambda, \times \mathbb{R}^{\text {Supp(i) }}\right)$ is a smooth manifold; the restriction of the natural projection to this manifold is proper. 
Proof. The main ingredient in the proof is simply the observation that, under the indecomposability assumption, the regular value theorem used in establishing the smooth sub-manifold property (See Balasko (1988), Chapter IV.) still works. Properness is straightforward. \#

Note that, if the indecomposability property is not satisfied, which incidentally turns out to be the case in the example where all the agents are restricted, a case met in Section 3 for $\lambda=1$, we cannot apply Theorem A.2 any more.

Theorem A.3. The set of Pareto optima for fixed total resources is diffeomorphic to $\mathbb{R}^{m-1}$ where $m$ denotes the total number of agents, Walrasian or quasi-Walrasian.

Proof. It suffices to reproduce the one for the purely Walrasian case. See, e.g., Balasko (1988), Section 5.2. \|

\section{B. PARETO OPTIMA AND THE WELFARE WEIGHTS}

The aim of this appendix is to recall some properties of Pareto optima of pure exchange economies and to give an explicit proof of a property used in this paper for which we have not been able to find appropriate references.

Using the notation of the paper, the set of Pareto optima $\bar{P}(H, \bar{r})$ is diffeomorphic to $\mathbf{R}^{m-1}$. (See, for example, Balasko $(1988,5.2 .4)$ and the discussion at the beginning of Section (5.2).) When preferences are defined by smooth strictly-concave utility functions (here denoted $v_{h}$ ), then Pareto optima can also be characterized as the solutions to the following maximization problem:

$$
\begin{array}{ll}
\text { Maximize } & \mathscr{L}\left(x_{1}, \ldots, x_{m}, \mu_{2}, \ldots, \mu_{m}\right)=v_{1}\left(x_{1}\right)+\mu_{2} v_{2}\left(x_{2}\right)+\cdots+\mu_{m} v_{m}\left(x_{m}\right) \\
\text { subject to } & \sum_{h} x_{h}=\bar{r}
\end{array}
$$

with $\mu_{2}, \mu_{3} \ldots, \mu_{m}>0$. For convenience, we write $\mu_{1}=1$. It is assumed from now on that each utility function is bounded from above, which can always be achieved by way of a concave, bounded from above, monotone transformation, a transformation that does not alter the set of Pareto optima $\bar{P}(H, \bar{r})$.

We also recall that since every utility function is defined on the whole commodity space, every indifference set $v_{h}^{-1}(c)$ is bounded from below for every $h$. (In Remark 1 , we discuss the changes brought by considering consumption spaces reduced to being the strictly positive orthant.) Given $\mu \in\{1\} \times \mathbf{R}_{++}^{\prime \prime}{ }^{-}$, this maximization problem has a solution and the solution is unique. Uniqueness is obvious. To establish existence, note that there exists $b_{h}$ for every $h$ such that $v_{h}\left(x_{h}\right) \leqq b_{h}$.

Let $x^{*}=\left(x_{h}^{*}\right)$ that satisfies $\sum x_{h}^{*}=\bar{r}$. Then, the problem of maximizing $\mathscr{L}\left(\left(x_{h}\right), \mu\right)$ subject to $\sum x_{h}=\bar{r}$ is equivalent to:

$$
\begin{array}{lc}
\text { Maximise } & \mathscr{L}\left(\left(x_{h}\right), \mu\right) \\
& \\
\text { subject to the two constraints } & \sum x_{h}=\bar{r} \\
& \mathscr{L}\left(\left(x_{h}\right), \mu\right) \geqq \mathscr{L}\left(\left(x_{h}^{*}\right), \mu\right) .
\end{array}
$$

The latter inequality is equivalent to

$$
v_{1}\left(x_{1}^{*}\right)+\mu_{2} v_{2}\left(x_{2}^{*}\right)+\ldots+\mu_{m} v_{m}\left(x_{m m}^{*}\right) \leqq v_{1}\left(x_{1}\right)+\mu_{2} v_{2}\left(x_{2}\right)+\ldots+\mu_{m} v_{m}\left(x_{m}\right)
$$

For $h$ arbitrary, one has

$$
v_{1}\left(x_{1}\right)+\ldots+\mu_{h} v_{h}\left(x_{h}\right)+\ldots+\mu_{m} v_{m}\left(x_{m}\right) \leqq b_{1}+\ldots+\mu_{h-1} b_{h-1}+\mu_{h} v_{h}\left(x_{h}\right)+\ldots+\mu_{m} b_{m}
$$

which, combined with the former inequality, yields

$$
\mu_{h} v_{h}\left(x_{h}\right) \geqq \mathscr{L}\left(x^{*}, \mu\right)-\sum_{k+h} \mu_{k} b_{k} .
$$

This implies that each $x_{h}$ is bounded from below. Combined with $\sum x_{h}=\bar{r}$, one is left with the problem of maximizing $\mathscr{L}(x, \mu)$ on a compact set, a problem with an obvious solution.

Define the map $\Psi: \mathbf{R}_{++}^{m-1} \rightarrow \bar{\Omega}$ that associates with $\left(\mu_{2}, \ldots, \mu_{m}\right) \in \mathbf{R}_{++}^{n-1}$ the solution to the maximization of $\mathscr{L}(x, \mu)$ subject to $\sum_{,} x_{h}=\bar{r}$. It is well-known that this map is a homeomorphism between $\mathbf{R}_{++}^{m-1}$ and the set of Pareto optima $\bar{P}(H, \tilde{r})$ : it establishes a continuous bijection, with a continuous inverse, between these two sets. See, e.g., Varian (1984, Section 5.7). On the other hand, the following property, which is necessary for our analysis, does not sem to have been stated in the literature. 
Proposition B.1. The map $\Psi: \mathbb{R}_{++}^{m-1} \rightarrow \bar{P}(H, \bar{r})$ is a diffeomorphism.

Proof. The inverse of $\Psi$ is the map $\Phi: \bar{P}(H, \bar{r}) \rightarrow \mathbb{R}_{++}^{m-1}$ that associates with the Pareto optimum $x=\left(x_{h}\right)$ the welfare weights $\mu_{2}, \ldots, \mu_{m}$. They can be defined by the formula

$$
\mu_{h}=\frac{\partial v_{1}\left(x_{1}\right)}{\partial x^{1}} / \frac{\partial v_{h}\left(x_{h}\right)}{\partial x^{1}} h=2, \ldots, m,
$$

which follows from the first-order conditions. Therefore the map $x=\left(x_{1}, \ldots, x_{m}\right) \in\left(\mathbb{R}^{l}\right)^{m} \rightarrow\left(\mu_{2}, \ldots, \mu_{m}\right)$ is smooth and its restriction to the sub-manifold $\bar{P}(M, \bar{r})$ is also smooth.

It remains to show that the map $\Phi: \mathbb{R}_{++}^{m-1} \rightarrow \bar{\Omega}$ itself is smooth. This will follow from the application of the implicit function theorem to the first-order conditions applied to the maximization problem defining the map $\Psi$. These first-order conditions take the form

$$
\left\{\begin{array}{c}
\sum_{h} x_{h}=\bar{r} \\
\mu_{h} D v_{h}\left(x_{h}\right)=D v_{1}\left(x_{1}\right), \quad h \in H \backslash\{1\}
\end{array}\right.
$$

It then suffices to check then that the Jacobian matrix of the first-order equation system with respect to the variables $x_{1}, \ldots, x_{m}$ is invertible. This matrix takes the form

$$
\left[\begin{array}{ccccc}
I & I & I & \cdots & I \\
-D^{2} v_{1}\left(x_{1}\right) & \mu_{2} D^{2} v_{2}\left(x_{2}\right) & 0 & \cdots & 0 \\
-D^{2} v_{1}\left(x_{1}\right) & 0 & \mu_{3} D^{2} v_{3}\left(x_{3}\right) & \cdots & 0 \\
\vdots & \vdots & \vdots & \ddots & \vdots \\
-D^{2} v_{1}\left(x_{1}\right) & 0 & 0 & \cdots & \mu_{m} D^{2} v_{m}\left(x_{m}\right)
\end{array}\right] .
$$

It is possible to subtract the first block column from the others, which yields.

$$
\left[\begin{array}{ccccc}
1 & 0 & 0 & \cdots & 0 \\
* & D^{2} v_{1}+\mu_{2} D^{2} V_{2} & D^{2} v_{1} & \cdots & D^{2} v_{1} \\
* & D^{2} v_{1} & D^{2} v_{1}+\mu_{3} D^{2} v_{3} & \cdots & D^{2} v_{1} \\
\vdots & \vdots & \vdots & \ddots & \vdots \\
* & D^{2} v_{1} & D^{2} v_{1} & \cdots & D^{2} v_{1}+\mu_{m} D^{2} v_{m}
\end{array}\right]
$$

This matrix has the same determinant as the matrix

$$
K=\left[\begin{array}{cccc}
D^{2} v_{1}+\mu_{2} D^{2} v_{2} & D^{2} v_{1} & \cdots & D^{2} v_{1} \\
D^{2} v_{1} & D^{2} v_{1}+\mu_{3} D^{2} v_{3} & \cdots & D^{2} v_{1} \\
\vdots & \vdots & \ddots & \vdots \\
D^{2} v_{1} & D^{2} v_{1} & \cdots & D^{2} v_{1}+\mu_{m} D^{2} v_{m}
\end{array}\right]
$$

This symmetric matrix is associated with the quadratic form ${ }^{\prime} X K X$ which is the sum of the quadratic forms defined by the matrices

$$
H=\left[\begin{array}{cccc}
D^{2} v_{1} & D^{2} v_{1} & \cdots & D^{2} v_{1} \\
D^{2} v_{1} & D^{2} v_{1} & \cdots & D^{2} v_{1} \\
\vdots & \vdots & \ddots & \vdots \\
D^{2} v_{1} & D^{2} v_{1} & \cdots & D^{2} v_{1}
\end{array}\right]
$$

and

$$
H_{2}=\left[\begin{array}{cccc}
\mu_{2} D^{2} v_{2} & 0 & \cdots & 0 \\
0 & 0 & \cdots & 0 \\
\vdots & \vdots & \ddots & \vdots \\
0 & 0 & \cdots & 0
\end{array}\right], \quad H_{3}=\left[\begin{array}{cccc}
0 & 0 & \cdots & 0 \\
0 & \mu_{3} D^{2} v_{3} & \cdots & 0 \\
\vdots & \vdots & \ddots & \vdots \\
0 & 0 & \cdots & 0
\end{array}\right],
$$


up to

$$
H_{m}=\left[\begin{array}{ccc}
0 & \cdots & 0 \\
\vdots & \ddots & \vdots \\
0 & \cdots & \mu_{m} D^{2} v_{m}
\end{array}\right] .
$$

Let the vector $X$ be decomposed into the block components $X_{2}, \ldots, X_{m}$ which yields the following decomposition of the row vector

$$
{ }^{\prime} X=\left({ }^{\prime} X_{2}, \ldots, X_{m}\right)
$$

Then, it follows readily from elementary matrix algebra that one has

$$
{ }^{\prime} X H_{h} X=\mu_{h}{ }^{\prime} X_{h} D^{2} v_{h} X_{h} .
$$

Furthermore, one has

$$
' X H X={ }^{\prime}\left(X_{2}+\ldots+X_{m}\right) D^{2} v_{1}\left(X_{2}+\ldots+X_{m}\right)
$$

as follows from the block structure of $H$. These computations imply the equality

$$
{ }^{t} X K X={ }^{\prime}\left(\sum_{h \geqq 2} X_{h}\right)\left(D^{2} v_{1}\right)\left(\sum_{h \geqq 2} X_{h}\right)+\sum_{h \geqq 2} \mu_{h}\left({ }^{t} X_{h} D^{2} v_{h} X_{h}\right) .
$$

Each term of this sum is $\leqq 0$ and at least one is $<0$ for $X \neq 0$. This implies that the matrix $K$ is associated with a negative definite quadratic form. Its determinant form is therefore $\neq 0$. $\|$

\section{ALTERNATIVE PROOF OF THE IDENTITY BETWEEN THE REGULAR ECONOMIES FOR THE UNRESTRICTED MODEL AND THE CERTAINTY ECONOMY}

The allocation $x=(x(\alpha), x(\beta))$ is an equilibrium allocation associated with $\lambda=0$ and $\omega=(\bar{\omega}, \bar{\omega})$ if and only if it is a solution of the following extended equation system.

$$
(*)\left\{\begin{array}{l}
D v_{h}\left(x_{h}(\alpha)\right)-\mu_{h} D v_{1}\left(x_{1}(\alpha)\right)=0, \quad h \neq 1, \\
D v_{h}\left(x_{h}(\beta)\right)-\mu_{h} D v_{1}\left(x_{1}(\beta)\right)=0, \quad h \neq 1, \\
\pi(\alpha) D v_{h}\left(x_{h}(\alpha)\right)\left(x_{h}(\alpha)-\bar{\omega}_{h}\right)+\pi(\beta) D v_{h}\left(x_{h}(\beta)\right)\left(x_{h}(\beta)-\bar{\omega}_{h}\right)=0, \quad h \neq 1, \\
\sum\left(x_{h}(\alpha)-\bar{\omega}_{h}\right)=0 \\
\sum\left(x_{h}(\beta)-\bar{v}_{h}\right)=0 .
\end{array}\right.
$$

Similarly, the allocation $\bar{x}$ is an equilibrium allocation associated with the certainty economy $\bar{\omega}$ if and only if it solves the equation system

$$
(\bar{*})\left\{\begin{array}{l}
D v_{h}\left(\bar{x}_{h}\right)-\bar{\mu}_{h} D v_{1}\left(\bar{x}_{1}\right)=0, \quad h \neq 1, \\
D v_{h}\left(\bar{x}_{h}\right)\left(\bar{x}_{h}-\bar{\omega}_{h}\right)=0, \\
\sum\left(\bar{x}_{h}-\bar{\omega}_{h}\right)=0 .
\end{array}\right.
$$

Let $\bar{A}=\{(\bar{x}, \tilde{\omega}) \mid \bar{x}$ is a solution of $(\bar{x})\}$. It follows from standard equilibrium theory that $\bar{A}$ is a smooth submanifold of $\Omega \times \bar{\Omega}$, that $\bar{\omega} \in \bar{\Omega}$ is a regular economy if and only if it is a regular value of the projection $(\bar{x}, \bar{\omega}) \rightarrow \bar{\omega}$ restricted to $\bar{A}$. Let

$$
A=\{(x(\alpha), x(\beta), \bar{\omega}) \mid x=(\alpha), x(\beta)) \text { is a solution of }\{\notin \subset \overline{\mathbf{\Omega}} \times \overline{\mathbf{\Omega}} .
$$

Then the map $(\bar{x}, \bar{\omega}) \rightarrow(\bar{x}, \bar{x}, \bar{\omega})$ from $\bar{\Omega} \times \bar{\Omega}$ into $(\bar{\Omega} \times \bar{\Omega}) \times \bar{\Omega}$ is a smooth embedding. Its restriction to the submanifold $A$ defines a smooth embedding denoted by $i$ from $\bar{A}$ into $(\bar{\Omega} \times \bar{\Omega}) \times \bar{\Omega}$. It follows from Lemma 2 that the image of this embedding is precisely the set $A$. This establishes that the set $A$ itself is a sub-manifold of 
$(\bar{\Omega} \times \bar{\Omega}) \times \bar{\Omega}$ diffeomorphic to $\bar{A}$ by a mapping $i$. The inverse of the map $i:(\bar{x}, \bar{\omega}) \rightarrow(\bar{x}, \bar{x}, \bar{\omega})$ is simply defined by the formula $j(\bar{x}, \bar{x}, \bar{\omega})=(\bar{x}, \bar{\omega})$. Therefore, the following diagram of smooth maps is commutative

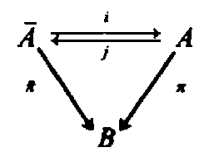

Let $(\bar{x}, \bar{\omega}) \in \bar{A}$ be a regular point of $\bar{\pi}$. This means that the tangent map $T_{\bar{x} \cdot \omega} \overline{\bar{\pi}}$ is onto. From $\bar{\pi}=\bar{\pi} \circ i$ follows that $T_{(\bar{x}, \sigma)} \bar{\pi}=T_{(\bar{x}, \bar{r}, \infty)} \pi \circ T_{(\bar{x}, \omega)} i$ is also onto, which implies that $T_{(\bar{x}, \bar{r}, \bar{\omega})} \pi$ is onto and, therefore, that $(\bar{x}, \bar{x}, \bar{\omega})$ is a

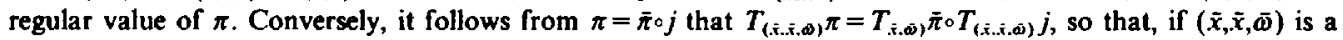
regular point of $\pi$, then $T_{(\bar{x}, \bar{x} . \Delta)} \pi$ is onto; this implies that $T_{(\bar{x} . \Delta)} \pi$ itself is onto and, consequently, that $(\bar{x}, \bar{\omega})$ is a regular point for $\bar{\pi}$. $\|$

Acknowledgments. Research support from the Swiss and U.S. National Science Foundations is gratefully acknowledged. We also acknowledge the support of the Department of Econometrics at the University of Geneva, The Center for Analytic Research in Economics and the Social Sciences at the University of Pennsylvania and the Center for Analytic Economics at Cornell University. The authors are grateful to the editors and to two referees for very helpful advice on the style and substance of this paper.

\section{REFERENCES}

ARROW, K. J. and HAHN, F. H. (1971) General Competitive Analysis. (San Francisco: Holden-Day).

AZARIADIS, C. and GUESNERIE, R. (1986), "Sunspots and cycles", Review of Economic Studies, 53, 725-736.

BALASKO, Y. (1983), "Extrinsic uncertainty revisited", Journal of Economic Theory, 31, 203-210.

BALASKO, Y. (1988) Foundations of the Theory of General Equilibrium. (Boston: Academic Press).

BALASKO, Y. (1990), "Equivalent general equilibrium theory", Journal of Economic Theory, 52, 18-44.

CASS, D. (1992), "Sunspots and incomplete financial markets: The general case", Economic Theory, 2, 341358.

CASS, D. and SHELL, K. (1983), "Do sunspots matter?", Journal of Political Economy, 91, 193-227.

GRANDMONT, J. M. (1989), "Local bifurcations and stationary sunspots", in W. A. Barnett, J. Geweke and K. Shell, (eds), Economic Complexity. (Cambridge: Cambridge University Press).

PRECHAC. C. (1988), "A Note on regular symmetric economies" (CAE Working Paper, Cornell University). (Journal of Economic Theory) (forthcoming).

VARIAN, H. (1984) Microeconomic Analysis, 2nd edition. (New York: Norton).

VILLANACCI, A. (1988), "A note on regular economies in sunspot models" (Preliminary paper, University of Pennsylvania). 